This is the author's version of a work that was accepted for publication in the Journal, Hydrometallurgy. Changes resulting from the publishing process, such as peer review, editing, corrections, structural formatting and other quality control mechanisms may not be reflected in this document. Changes may have been made to this work since it was submitted for publication. A definitive version was subsequently published in the Journal, Hydrometallurgy, Volumes 119-120, May 2012, Pages 47-54.

http://doi.org/10.1016/j.hydromet.2012.02.017 


\title{
Simulation of iron oxide/silica precipitation in the Paragoethite Process for the removal of iron from acidic zinc leach solutions
}

${ }^{1}$ Laurence G. Dyer, ${ }^{1}$ William R. Richmond, ${ }^{2}$ Phillip D. Fawell

${ }^{1}$ Curtin University, Parker Centre for Integrated Hydrometallurgy Solutions

${ }^{2}$ CSIRO Process Science and Engineering, Parker Centre for Integrated

Hydrometallurgy Solutions

Corresponding Author Contact:

Email: laurence.dyer@ubc.ca

Ph: 011-1-604-827-5070

Fax: 011-1-604-822-3619

Department of Materials Engineering

University of British Columbia

309-6350 Stores Road

Vancouver, BC. V6T 1Z4

\begin{abstract}
An investigation of the simultaneous precipitation of iron oxide and silica species from acidic solutions was conducted, simulating the Paragoethite Process, an ironremoval stage employed in zinc hydrometallurgy. Laboratory-based continuous crystallisation experiments were carried under the primary conditions employed in industry, with $\mathrm{pH}$ (at $85^{\circ} \mathrm{C}$ ) maintained at 2.65 , the combined $\mathrm{Fe}$ and $\mathrm{Si}$ concentration at $0.1128 \mathrm{M}$ (unless otherwise stated) and $\mathrm{Si}: \mathrm{Fe}$ ratios varied from 0 to 0.43 . The crystal structure of the residues produced was characterised, with the extent of iron and silicate removal quantified and properties relating to aggregate structure also measured.
\end{abstract}


It is shown that the degree of silica polymerisation prior to the precipitation reaction dictates the mechanism of co-precipitation and thereby the properties of the residue. In the presence of polymerised silica, less dense aggregates of finer mean particle size are formed, which has negative implications for dewatering. Conversely, where silica is allowed to polymerise prior to the iron precipitation reaction, a greater proportion of silica is removed from solution. Close control of silica polymerisation in the feed liquor is therefore required to reach a compromise between its beneficial and detrimental influences, and to thereby obtain optimal performance.

\section{Introduction}

\subsection{Zinc hydrometallurgy}

Iron is often the primary impurity in zinc-bearing ores and any acidic leaching of zinc will inevitably also lead to at least partial dissolution of iron phases. Electrowinning is favoured for the recovery of pure zinc from the leach solutions, but this first requires the removal of iron. There are numerous zinc hydrometallurgical processes known in which this removal is achieved via a precipitation step involving an increase in the liquor pH (Dutrizac, 1987; Loan, 2006a).

While zinc hydrometallurgical processes around the world have many similarities, variations in ores, flowsheets and operating conditions makes each process unique and in particular provide specific challenges in the iron removal stage (Cornell, 1996). This study focuses on the Nyrstar Metals Ltd. Hobart Smelter in Australia, which has been in operation for close to a century. Zinc concentrates processed at the Smelter contain iron-substituted sphalerite $(\mathrm{Zn} / \mathrm{FeS})$ and gangue minerals such as pyrite $\left(\mathrm{FeS}_{2}\right)$, pyrrhotite $\left(\mathrm{Fe}_{(1-\mathrm{x})} \mathrm{S}\right)$, hematite $\left(\mathrm{Fe}_{2} \mathrm{O}_{3}\right)$ and chalcopyrite $\left(\mathrm{CuFeS}_{2}\right)$. Iron constitutes between 3 and $18 \%$ of the concentrates. Phase transformation occurs under roasting, forming some franklinite $\left(\mathrm{ZnFe}_{2} \mathrm{O}_{4}\right)$ and hematite. As franklinite is only soluble under extreme $\mathrm{pH}$ conditions, it can be separated from zincite by neutral leaching. Franklinite and other sparingly soluble minerals are dissolved in hot and strong acid leaching stages $\left(90-95^{\circ} \mathrm{C}, 70-130 \mathrm{~g} \mathrm{~L}^{-1} \mathrm{H}_{2} \mathrm{SO}_{4}\right)$ (Loan, 2004). 
Silicon appears in all the ores processed at the Hobart Smelter, in proportions that vary depending on the site from which the raw materials are obtained. The effect of increased levels of silicate in plant liquors remains poorly understood, but there is strong evidence of detrimental impacts on dewatering and zinc electrowinning (Cooper, 1998). If the silica is not satisfactorily removed, other measures would need to be implemented to maintain final product purity (Takemori, 1993), which would require more time and capital. This study was aimed at clarifying the impact of liquor silicate - is silicate effectively removed during iron precipitation, how does this affect residue properties, and can its presence within a residue influence zinc losses during the iron removal process?

\subsection{The Paragoethite Process}

For many years the Hobart Smelter produced jarosite $\left((\mathrm{K}, \mathrm{Na}, \mathrm{Pb}) \mathrm{Fe}_{3}\left(\mathrm{SO}_{4}\right)_{2}(\mathrm{OH})_{6}\right)$ as an iron oxide by-product (Arregui, 1979; Kershaw, 1995). While it enabled excellent zinc recovery and purity, the jarosite process was associated with large amounts of harsh scale and the dewatering and disposal of the precipitate was a major concern. Such issues led to the introduction of the Paragoethite Process currently in operation at the smelter. The process takes its name from the predominantly iron oxy-hydroxide residue formed that was initially thought to closely resemble goethite (Claassen, 2002).

Within the Paragoethite Process, the leach liquor $\left(85^{\circ} \mathrm{C}, \sim 16 \mathrm{~g} \mathrm{~L}^{-1} \mathrm{Fe}^{3+}, \sim 160 \mathrm{~g} \mathrm{~L}^{-1}\right.$ $\mathrm{ZnSO}_{4}$ ) is fed continuously into the reactor at a rate chosen to promote dilution, and calcine (predominantly zinc oxide) slurry is added to achieve the $\mathrm{pH}_{33}$ of 3.5 (Loan, 2006a). This value is highly important, as variation outside a small $\mathrm{pH}$ range results in the precipitation of solids with poor solid-liquid separation properties (Schwertmann, 2000).

There are both positive and negative aspects to this process. An advantage of the residue is that its high surface area and strongly positive surface charge at the reaction $\mathrm{pH}$ provides a high affinity for adsorption of anionic impurities, such as arsenic and silicates (Richmond, 2004; Sigg, 1981). A significant degree of cation adsorption also occurs, removing impurities such as sodium and lead, irrespective of the surface 
charge. It retains relatively low levels of sulphate and the operating costs are low, but there are greater zinc losses than associated with the jarosite process. Poor solid-liquid separation can be a major impediment to plant operation, making the residue unfit for transport or further refining. Presently, fine adjustments in reaction conditions such as $\mathrm{pH}$ are made to achieve more favourable residue qualities, although not always with success.

The complexity of the reaction matrix produces a residue consisting of a plethora of species, the identification of which was the subject of a comprehensive examination by Loan (Loan, 2004). The residue contains undissolved calcine materials, such as franklinite, willemite and sphalerite, as well as the precipitated iron oxide phases. The primary product of the precipitation reaction is 6-line ferrihydrite, which accounts for approximately $50 \%$ of the residue (Loan, 2006a). However, other iron oxides present can include goethite, schwertmannite and jarosite.

\subsection{Approach}

The work by Loan and coworkers (Loan, 2004; Loan, 2006a) did not consider silicates that may co-precipitate with iron in any detail. While it provides the foundation for the present study (in particular highlighting the need for continuous crystallisation for residue preparation), it offers limited insights towards explaining the variability in residue behaviour in plant operation (e.g. thickening, zinc uptake, level of silica removal).

The first stages of this work focused on using small-scale continuous preparations (reactor volume $0.11 \mathrm{~L}$ ) to prepare residues for characterisation that provided insights on fundamental aspects of the residue formation process (Dyer, 2010; Dyer, 2012). Structural information, elemental composition and surface chemistry allowed the association between the iron oxides and silica to be determined. The implications for dewatering could not be readily elucidated from these studies, primarily because the small masses of residue formed generally only provided sufficient sample for crystallographic and microscopic structural characterisations. 
It was shown by Dyer et al. (Dyer, 2010) that the primary anions present in solution play an important role in the behaviour of the co-precipitate formed. Plant liquors contain high concentrations of sulphate, which was revealed to inhibit surface interactions between the iron oxide phase and silica solution molecules (primarily monosilicic acid). This is due to the strong association between sulphate and ferrihydrite. Experiments conducted in nitrate media did not show the same competitive adsorption, as nitrate displays a much weaker binding affinity. A comparison of residues formed in the presence of both sulfate and nitrate dominated solutions is therefore of significant interest.

A larger continuous crystallisation apparatus than detailed in Dyer et al. (Dyer, 2010) was designed to provide sample volumes great enough for physical property measurements. Crystallisation experiments were conducted at similar $\mathrm{pH}$ and temperature to plant conditions, using analytical grade reagents without the complex matrix of impurities present in plant liquors, to allow insight into specific residue properties. As 6-line ferrihydrite is the primary product of the Paragoethite Process, the operating conditions were designed predominantly to reflect the process, but were also chosen to substantially favour ferrihydrite over other iron oxide phases.

This article presents data concerning the impact of silica on ferrihydrite behaviour. It addresses crystal structure and phase, iron and silicate removal and properties relating to aggregate structure and formation. The degree of silica polymerisation in feed solutions prior to the iron precipitation reaction has been considered. Possible industrial impacts are derived from aggregate and surface data obtained from systems closely resembling plant conditions.

\section{Experimental}

\subsection{Crystallisation}

The crystallisation apparatus consisted of a $3 \mathrm{~L}$ (reaction volume $=2.5 \mathrm{~L}$ ), baffled, stainless steel reactor $(150 \mathrm{~mm}$ internal diameter, $175 \mathrm{~mm}$ internal height), continuously fed by two solutions: iron (III)/silicate solution and $8 \mathrm{M}$ sodium 
hydroxide solution, the latter to maintain $\mathrm{pH}$ at 2.65 at $85^{\circ} \mathrm{C}$. The reactor was continually agitated by an impeller (three $45^{\circ}$ angled blades, $500 \mathrm{rpm}$ ) positioned $20 \mathrm{~mm}$ from the bottom of the reactor to ensure proper mixing, while a water jacket maintained the solution temperature.

Table 1 summarises the range of experimental conditions examined and the codes by which the experiments and residue solids are identified. For the codes, the first letter (i.e. $\underline{\mathrm{S}}$-20-P1) refers to the primary anion present ( $\mathrm{S}$ is sulfate, $\mathrm{N}$ is nitrate); the first number $(\mathrm{S}-\underline{20}-\mathrm{P} 1)$ refers to the molar percentage of $\mathrm{Si}$ with respect to $\mathrm{Si}+\mathrm{Fe}$ in the feed solution; the final letter (S-20-르) refers to the state of silica in the feed (P for polymeric, $\mathrm{M}$ for monomeric); if a number accompanies the last letter (S-20-P 2 ), then there are multiple experiments that fulfil these criteria. The combined $\mathrm{Si}+\mathrm{Fe}$ concentration was $0.1128 \mathrm{M}$ for all experiments other than S-20-P2, which was less concentrated $(\mathrm{Si}+\mathrm{Fe}=0.0894)$. The $\mathrm{Si}: \mathrm{Fe}$ ratios studied ranged from 0 to 0.43 .

The term 'residence time' in these experiments refers to the time required for one full reaction volume $(2.5 \mathrm{~L})$ to pass through the system under continuous operation. The flow rates were adjusted to produce a 45 minute residence time. Based on previous work (Loan, 2006b), six residence times were required to reach a steady-state (equilibrium) in the formation of ferrihydrite. The slurry removal rate $\left(\sim 3.3 \mathrm{~L} \mathrm{~h}^{-1}\right)$ matched the combined rate for the feed solutions, thereby maintaining a constant volume of $2.5 \mathrm{~L}$ in the reactor. Watson Marlow 505J pumps were used for the iron feed solution and product removal flows, while a Gilson Minipuls 3 pump was used to accommodate the lower flow requirement for the sodium hydroxide solution.

The iron and silicate portions of the feed were dissolved separately. This is due to an increase in $\mathrm{pH}$ upon the dissolution of sodium metasilicate, as the hydrolysis reaction releases hydroxide ions. The silicate solution required acidification before being combined with the ferric sulfate so as not to induce iron precipitation in the feed. The appropriate acid (sulphuric acid in sulphate media, nitric acid in nitrate media) was used to adjust the $\mathrm{pH}$ of the silicate solution below $\mathrm{pH} 2$. 
Preparation of the samples in which silica polymerisation was deliberately induced in the feed required the silicate source to be dissolved at high concentration to achieve a high order of silica supersaturation. The entire mass of silicate source was dissolved in MilliQ ${ }^{\circledR}$ water, using $0.5 \mathrm{~L}$ of water for Si:Fe ratios of 0.053 and 0.11 , and $1 \mathrm{~L}$ for ratios of 0.25 and 0.43 . Any smaller volumes of water would have produced a concentration above the gelation point of silica once acidified, inducing rapid formation of a gel. The solubility of silica is much reduced at low $\mathrm{pH}$ and therefore the other purpose of acidifying the solution is to create supersaturation with respect to silica. After the solution was acidified, it was left to stand for ten minutes before dilution into the rest of the feed solution, which was then made up to its final volume. An experiment with a $\mathrm{Si}: \mathrm{Fe}$ ratio of 0.43 was also conducted without inducing polymerisation in the feed (S-30-M) for the purpose of comparison.

Table 1: Reagent concentrations for each experiment and whether silica polymerisation was induced in the feed solution.

\begin{tabular}{|c|c|c|c|}
\hline System ID & Fe conc. $\left(\mathrm{g} \mathrm{L}^{-1}\right)$ & Si conc. $\left(\mathrm{g} \mathrm{L}^{-1}\right)$ & Polymerisation in feed \\
\hline S-0 & 6.3 & 0 & N/A \\
\hline S-5-P & 6.0 & 0.16 & Yes \\
\hline S-10-P & 5.7 & 0.32 & Yes \\
\hline S-20-P1 & 5.0 & 0.63 & Yes \\
\hline S-30-P & 4.4 & 0.95 & Yes \\
\hline S-20-P2 & 4.0 & 0.50 & Yes \\
\hline S-30-M & 4.4 & 0.95 & N/A \\
\hline N-0 & 6.3 & 0 & No \\
\hline N-5-M & 6.0 & 0.16 & No \\
\hline N-10-M & 5.7 & 0.32 & No \\
\hline N-30-M & 4.4 & 0.95 & \\
\hline
\end{tabular}

\subsection{Analytical Process}

Solids samples for XRD were filtered using $0.45 \mu \mathrm{m}$ membranes, washed with MilliQ ${ }^{\circledR}$ water and oven-dried at $60^{\circ} \mathrm{C}$ for several days. The measurements were conducted on a Phillips X'Pert powder diffractometer using a Co long fine focus tube. 
ICP analysis was performed on supernatant solutions filtered through a $0.45 \mu \mathrm{m}$ membrane. The data was collected using an ICP-AES Vista instrument. Slurry subsamples were dialysed with MilliQ ${ }^{\circledR}$ water for 6 days (water refreshed daily) prior to zeta potential measurements on a Malvern Zetanano DLS particle sizer. The $\mathrm{pH}$ was adjusted in these samples using either dilute nitric acid or sodium hydroxide.

Particle size distributions (PSDs) were obtained on slurry sub-samples using a Malvern Mastersizer 2000. The diameter below which $10 \%$ and $50 \%$ of the particles exist ( $D_{10}$ and $D_{50}$, respectively) along with the mean particle diameter $\left(D_{4,3}\right)$ are presented to represent the sample PSDs. Fractal dimension values for aggregated particles can be extracted from PSD data obtained from light scattering by plotting the $\log$ of the intensity from each detector against the log of the detector angle, although not all detectors are considered in the calculation. A region of linear decrease in the $\log$ of the intensity at increasing angles can be discerned in the plot. The negative of the gradient of this region is the fractal dimension. This technique has proven very useful in pure, mondispersed systems (Selomulya, 2001; Zhou, 2006). The complexity of the systems dealt with in this investigation leads to significant uncertainty, and as a consequence the analysis is used to only provide evidence of trends, as opposed to absolute fractal dimension values.

Solid samples for physical property determinations were collected on a $0.22 \mu \mathrm{m}$ filter membrane, washed with MilliQ ${ }^{\circledR}$ water and dried at $100^{\circ} \mathrm{C}$. Analyses for particle density and surface area were performed using a Quantichrome Multipycnometer MVP-1 and Micromeritics Tristar 3000, respectively. Rheological measurements were conducted on slurry samples, concentrated by centrifugation and decantation, using a Haake Rheometer VT 550 and either an FL-1000 or Fl-100 vane, depending on the volume of sample available. The data was compiled using Thermo-Haake Rheowin Pro software. 


\section{Results and Discussion}

\subsection{Silica Removal}

The primary issue relating to silica as a process impurity is the efficiency with which it can be removed. At trace levels it is simply adsorbed by the residue in the same vein as other impurities, such as arsenic. However, at higher concentrations, simple adsorption will not be effective and polymerisation of the silica must also be considered.

Table 22 displays the proportions of iron and silicon remaining in solution after the co-precipitation reaction for experiments conducted in sulphate media (this effect was not examined in the presence of nitrate).

Table 2: Elemental concentrations remaining in supernatant solutions extracted from steady-state samples from each experiment in sulphate media.

\begin{tabular}{|c|c|c|c|c|}
\hline System & Fe in Liquor (ppm) & Fe Precipitated (\%) & Si in Liquor (ppm) & Si Precipitated (\%) \\
\hline S-0 & 10.3 & 99.8 & - & - \\
\hline S-5-P & 2.7 & $\sim 100$ & 92.0 & 40.1 \\
\hline S-10-P & 2.9 & $\sim 100$ & 108 & 62.1 \\
\hline S-20-P1 & 11.9 & 99.8 & 261 & 57.1 \\
\hline S-30-P & 13.7 & 99.7 & 263 & 68.9 \\
\hline S-20-P2 & 0.6 & $\sim 100$ & 131 & 72.9 \\
\hline S-30-M & 22.7 & 99.4 & 648 & 31.6 \\
\hline
\end{tabular}

The values obtained for S-30-M are consistent with previous experiments (Dyer, 2010) involving co-precipitate preparation in sulphate media without silica polymerisation in the feed solution. All reactions in which polymerisation was induced, regardless of initial concentrations, precipitated a greater percentage of silica. This demonstrates that silica polymerisation occurring in feed liquors prior to the precipitation reaction increases the efficiency of the silica removal with the iron product. Also, as one might expect, greater silicate concentrations in the feed generally result in a greater percentage of silica precipitated. The main variation from this trend was the lower than expected value for S-20-P1, which can be attributed to 
the difference in the silicate saturation level at which polymerisation was induced. The sodium metasilicate used in S-20-P1 was dissolved in twice the volume of water than that of S-10-P to avoid gelation. This greater volume led to a lower relative silicate concentration, and thus a lower degree of saturation.

The presence of sulphate as the primary anionic constituent inhibits the removal of silica with iron, whereas nitrate does not (Dyer, 2010). The use of nitrate in plant liquors is not a practical consideration in zinc hydrometallurgy, therefore enhancing silica polymerisation in sulphate process liquors prior to the iron precipitation step is desirable in terms of effective impurity removal. However, the impact of this polymerisation on the physical properties of the co-precipitates is another factor to be considered.

\subsection{Ferrihydrite Crystallinity}

It has been shown that the presence of soluble silicate during ferrihydrite precipitation decreases the product crystallinity and retards the formation of more crystalline phases, such as goethite (Carlson, 1981; Cornell, 1996; Dyer, 2010). The XRD data presented in Figure 1 shows a marked difference in the structural attributes of the precipitates prepared in experiments $\mathrm{S}-0$ and $\mathrm{S}-30-\mathrm{M}$. Reflections attributed to goethite are visible in the silica-free system but are absent in S-30-M. In addition, there is an obvious broadening of the ferrihydrite peaks in the S-30-M pattern. A similar trend in peak broadening can be seen throughout the patterns from the nitrate system samples (figure 2). 


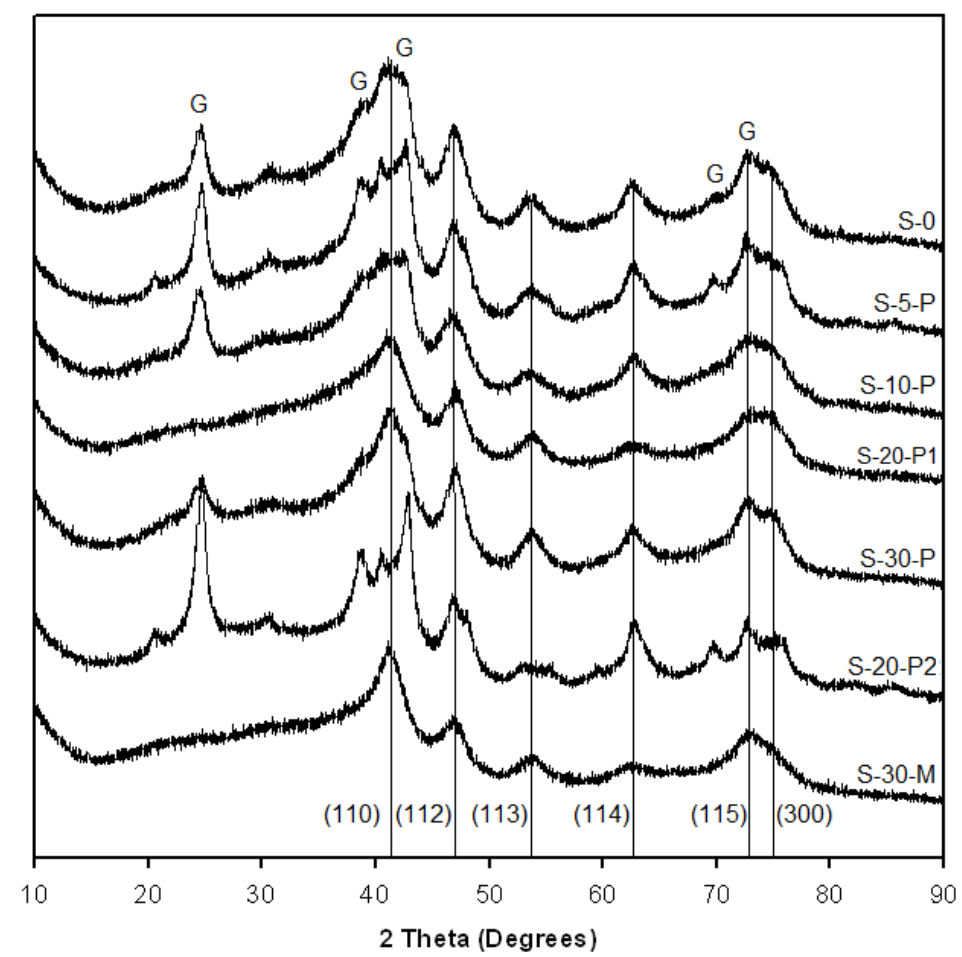

Figure 1: XRD patterns obtained from samples of experiments conducted in the presence of sulphate ions. Ferrihydrite peaks are marked by the vertical lines and provided with reflection planes; (G) refers to the goethite peaks, which may occur at the same angle as ferrihydrite peaks. With the exception of the pattern obtained from S-20-P2 the silica content in the precipitate increases from top to bottom.

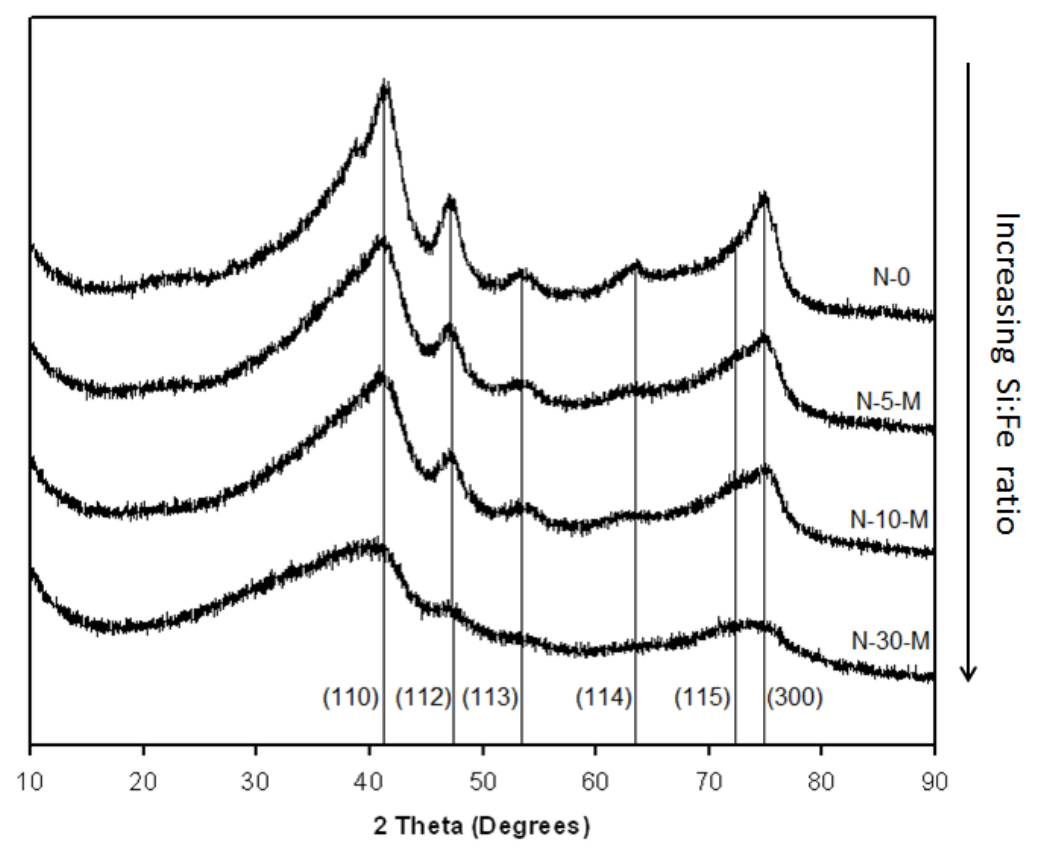

Figure 2: XRD patterns obtained from samples of all experiments conducted in the presence of nitrate ions. Ferrihydrite peaks are marked by the vertical lines and provided with reflection planes.

Conversely, there is evidence of goethite in the patterns of almost all of the sulphate system samples with silica polymerisation in the feed (S-5-P, S-10-P, S-20-P2 and 
S-30-P) and there is also no significant ferrihydrite peak broadening in these patterns (figure 1). S-20-P1 is the exception from this group, again due to supersaturation at which the silicate portion of the feed solution was prepared. The pattern obtained from S-20-P2 displays the strongest goethite reflections, indicating significantly more goethite than any other sample. This is due to the lower initial iron concentration resulting in a slower hydrolysis rate and thus a more crystalline product.

This suggests a diminished capacity of polymerised silica to influence the particle size of the ferrihydrite and inhibit the transformation to more crystalline material. Polymerised silica would have fewer interactions with the forming ferrihydrite, as individual silicate molecules would be non-existent, or at least less prolific, having been consumed during polymerisation. Polymers and small particles or colloids would be expected to interact with the ferrihydrite surface differently to monomeric silica. There would be a significantly reduced number of collisions between ferrihydrite and silica species, leading to fewer overall binding events. In addition, rather than individual silicic acid molecules adsorbing onto a large proportion of the binding sites across the ferrihydrite surface, large polymers and particles adsorbed on the surface may interfere with the approach of others species and obscure areas of the surface.

In comparison with monomeric silica, this would lead to large amounts of silica attached to ferrihydrite particles but being bound at relatively few sites. Such an association would be unlikely to restrict the growth of ferrihydrite particles or provide the same structural stabilising effect, therefore leaving the ferrihydrite vulnerable to transformation. This explains the sharper, more distinct ferrihydrite peaks and the occurrence of reflections attributed to goethite and hematite. The implication is that the pre-polymerised silica appears in the co-precipitate as discrete particles, whereas the monomeric silica adsorbs and then polymerises on the ferrihydrite surface. 


\subsection{Physical Properties}

\subsubsection{Aggregation}

Table 3 contains a summary of physical property measurements that are pertinent to surface behaviour and, on a practical level, to dewatering through gravity thickening or filtration.

Table 3: Measurements of physical properties of the co-precipitates for both sulphate and nitrate systems.

\begin{tabular}{|c|c|c|c|c|c|}
\hline Sample & $\begin{array}{c}\text { Fractal } \\
\text { Dimension }\end{array}$ & $\begin{array}{c}\text { Surface Area } \\
\left(\mathrm{m}^{2} \mathrm{~g}^{-1}\right)\end{array}$ & $\mathrm{d}_{10}(\mu \mathrm{m})$ & $\mathrm{d}_{50}(\mu \mathrm{m})$ & $\mathrm{d}_{4,3}(\mu \mathrm{m})$ \\
\hline S-0 & 2.07 & 132 & 9.28 & 16.3 & 16.8 \\
\hline S-5-P & 1.81 & 109 & 3.22 & 17.1 & 17.4 \\
\hline S-10-P & 1.72 & 150 & 2.17 & 15.7 & 16.2 \\
\hline S-20-P1 & 1.87 & 249 & 1.52 & 8.56 & 8.79 \\
\hline S-30-P & 1.45 & 255 & 0.90 & 6.74 & 7.20 \\
\hline S-20-P2 & 2.18 & 210 & 9.71 & 20.7 & 22.0 \\
\hline S-30-M & 1.81 & 276 & 6.08 & 13.2 & 13.5 \\
\hline N-0 & 1.55 & 171 & 1.12 & 7.44 & 8.42 \\
\hline N-5-M & 2.28 & 246 & 1.39 & 5.04 & 8.33 \\
\hline N-10-M & 1.64 & 277 & 1.67 & 12.3 & 12.8 \\
\hline N-30-M & 1.73 & 370 & 2.65 & 17.5 & 18.4 \\
\hline
\end{tabular}

The fractal dimension for each co-precipitate was investigated as it is indicative of the manner in which particles in an aggregate are packed (Jullien, 1987). As was stated earlier, the complexity of the systems studied and the broad distribution of particle sizes means that the estimated fractal dimension values should only be used to provide relative comparisons.

The higher the fractal dimension, the more compact the particles are bound together; aggregates with lower values exhibit more open structures and are likely to contain more weakly associated particles (Meakin, 1988). Critically, at a high fractal dimension the aggregates will contain less associated water and at equivalent sizes will settle faster. The subsequent flocculation of such aggregates for gravity 
thickening is complicated by a number of factors, but in general a higher fractal dimension for the precipitated solids is highly desirable as it reduces the effective volume fraction of the cluster aggregates formed with polymer flocculants, thereby lowering the optimum solids concentration for flocculation and the need for solids dilution prior to flocculant application (Fawell, 2009; Owen, 2008). Other practical implications include greater volumetric throughput, higher thickener underflow density and potentially reduced rake torque.

The impact of polymerisation in the silica feed is apparent in the fractal dimensions and particle size distribution statistics for the residue solids (Table 3). Where polymerised silica is present, the fractal dimension of the residue decreases with an increasing Si:Fe ratio ( $\mathrm{S}-20-\mathrm{P} 1$ is again the exception to this trend for the reasons already discussed).

While the fractal dimension value obtained for S-30-M (1.81) is lower than that obtained in the absence of silicate (residue S-0, 2.07), it is much greater than that for S-30-P (1.45), suggesting that monomeric silica has a substantially smaller influence on the aggregation process than in its pre-polymerised form. More tightly packed aggregates obtained from the presence of monomeric silica would likely display more desirable solid/liquid handling behaviour.

The high fractal dimension of the S-20-P2 residue is attributable to the relatively large proportion of goethite in the sample. This goethite is the result of a slower hydrolysis rate, induced by a lower iron concentration in the feed.

Residue N-0 has a much lower fractal dimension than S-0. As more crystalline phases such as goethite and hematite display a greater fractal dimension, the low value associated with N-0 may be due to the lack of such phases in the precipitate. Our previous work has shown that sulphate ions decrease the surface charge of ferrihydrite at the reaction $\mathrm{pH}$ (Dyer, 2010). Therefore, the difference in fractal dimension may also be related to the passivation of the surface charge by sulphate ions, providing less resistance to aggregation. 
The introduction of silicate in the feed in nitrate media appears to have a positive influence on aggregation; although all co-precipitates displayed higher fractal dimensions then the silica-free sample, the greatest increase was observed in the value obtained from the N-5-M sample. Of course, the nitrate systems were only studied with monomeric silica, which had a reduced impact in the sulphate systems relative to its polymerised form. Why the N-5-M sample diverged from expectations remains unclear. Interestingly, N-5-M also had a lower mean aggregate size, which does not normally correlate with a high fractal dimension.

Excluding N-5-M, size distribution data did generally relate to fractal dimension values as expected (higher fractal dimension equating to larger sizes). The presence of polymerised silica in the feed produces smaller, more sparsely-bound aggregates, become progressively smaller at greater initial silicate concentrations. The divergence of the S-20-P2 values from the general observed trend is again attributable to large proportions of goethite. Monomeric silica either favours the formation and stability of larger aggregates (as in the case of the nitrate experiments) or is at least less detrimental than polymerised silica (S-30-M).

Rheological measurements on particulate systems may also be used to provide an indication of aggregation under higher solids conditions that may be of practical relevance (i.e. for thickener underflow or filtration). None of the slurries as formed were at a high enough solids concentration to produce a detectable yield stress from vane measurements. It was therefore necessary to apply centrifugation and decantation to concentrate the samples. Even after centrifugation, S-0, S-5-P and S-10-P gave negligible yield stress values $(<10 \mathrm{~Pa})$ at their respective highest achievable solids concentrations (506, 767 and $632 \mathrm{~g} \mathrm{~L}^{-1}$, respectively). In contrast, S-20-P1 (748 $\left.\mathrm{g} \mathrm{L}^{-1}\right)$ and S-30-P $\left(655 \mathrm{~g} \mathrm{~L}^{-1}\right)$ provided maximum values of 42.0 and $346 \mathrm{~Pa}$, respectively. Despite the variation is solids concentrations, a clear trend is evident towards greater yield stress values at higher Si:Fe ratios, which reflects the decreasing mean aggregate size. The yield stress for S-30-M was negligible even at $772 \mathrm{~g} \mathrm{~L}^{-1}$, suggesting the above trend is related to the presence and proportion of polymerised silica and its influence on the mean aggregate size. 
The most startling result from the rheology measurements related to S-20-P2, for which the highest solids concentration that could be attained was only $291 \mathrm{~g} \mathrm{~L}^{-1}$, and yet this still gave a high yield stress (194 Pa). This result is likely another symptom of high proportions of goethite; the mixture of morphologies (dense, rigid goethite rods combined with the open polygonal ferrihydrite aggregates) may alter the interactions between aggregates and therefore impede dewatering.

For the nitrate system, the yield stress values at maximum solids concentration were 478, 59, 91 and $61 \mathrm{~Pa}$ for residues $\mathrm{N}-0$ (658 $\left.\mathrm{g} \mathrm{L}^{-1}\right), \mathrm{N}-5-\mathrm{M}\left(346 \mathrm{~g} \mathrm{~L}^{-1}\right), \mathrm{N}-10-\mathrm{M}$ $\left(470 \mathrm{~g} \mathrm{~L}^{-1}\right)$ and N-30-M (393 $\left.\mathrm{g} \mathrm{L}^{-1}\right)$, respectively. The interpretation of such results is not straight-forward, but the implication is that, assuming a conventional yield stress response to solids concentration for the $\mathrm{N}-0$ residue, at solids concentrations below $500 \mathrm{~g} \mathrm{~L}^{-1}$, its yield stress would in all likelihood have been $<10 \mathrm{~Pa}$. Therefore the presence of silicate in the nitrate system appears to enhance aggregation, consistent with the size information in Table 3 (i.e. the observed increase in size is a consequence of aggregation, not an increase in primary particle size).

\subsubsection{Co-precipitate Surface}

The adsorptive properties of the residue are key to both the purity of the zinc-bearing solution and to minimising the levels of zinc adsorption. The surface charge bears great importance as to which solution species are likely to be adsorbed at reaction $\mathrm{pH}$. We have previously shown that the presence of negatively charged sulphate ions, which bind strongly with the ferrihydrite surface, decreases the magnitude of the positive zeta potential, and shifts the iso-electric point (IEP) itself to lower $\mathrm{pH}$ values (Dyer, 2010).

The plots of zeta potential as a function of $\mathrm{pH}$ in Figure 3Figure 3 display the decrease in IEP due to the greater $\mathrm{Si}: \mathrm{Fe}$ ratios in the feed solution, for residue samples when silica polymerisation is induced in the feed. Even though the ferrihydrite-silica association is different based on the degree of silica polymerisation (polymerised silica will not form the same surface layer on ferrihydrite) there is still a significant impact on the average surface charge. 


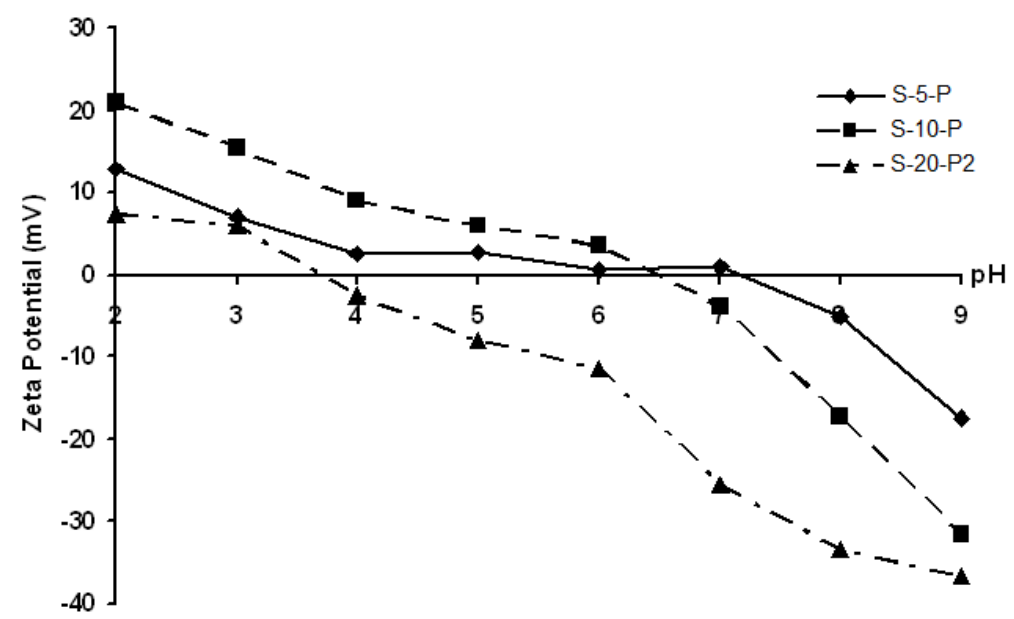

Figure 3: Plots of the zeta-potential against $\mathrm{pH}$ for the S-5-P, S-10-P and S-20-P2 samples, displaying the decrease in IEP due to greater $\mathrm{Si}: \mathrm{Fe}$ ratios.

As high sulphate concentrations are a feature of the Paragoethite Process, the influence of the ion would already be imposed on the residue. However, the decline in IEP with increasing silicate concentrations in the feed may pose a concern. As the IEP decreases consistently with greater proportions of silica, the net surface charge may then be very small or zero at reaction $\mathrm{pH}\left(\mathrm{pH}_{33}=3.6\right)$ depending on the silica content. Decreasing the IEP with high proportions of silica would provide less resistance to cation adsorption than a residue produced during the processing of lower silicate ores, which could increase zinc losses.

Within plant residues, it has been shown that larger relative proportions of the total zinc losses can be associated with coarse size fractions within the residue solids, as opposed to fines (D Palmer, personal communication, 2008). To determine whether this was related to adsorption, surface charge measurements were conducted on samples containing material with significantly different mean particle sizes. The separation was achieved by allowing the slurry to settle for 10 minutes and sampling the slightly turbid supernatant with a Pasteur pipette, with the remaining suspended particles therefore representing the smallest size fraction available.

The surface charge of the suspended particles as a function of $\mathrm{pH}$ was compared with those of the bulk precipitates from samples produced in S-10-P and S-20-P2 (Figure 4). There was no noticeable difference in the zeta potentials measured from the bulk sample and sub-sampled fines. Therefore it appears there was little variation in the 
surface species present on the co-precipitate solids in relation to the particle size. This result would suggest that it is unlikely that adsorption behaviour would differ significantly enough to produce large inconsistencies in zinc content between particle size fractions.
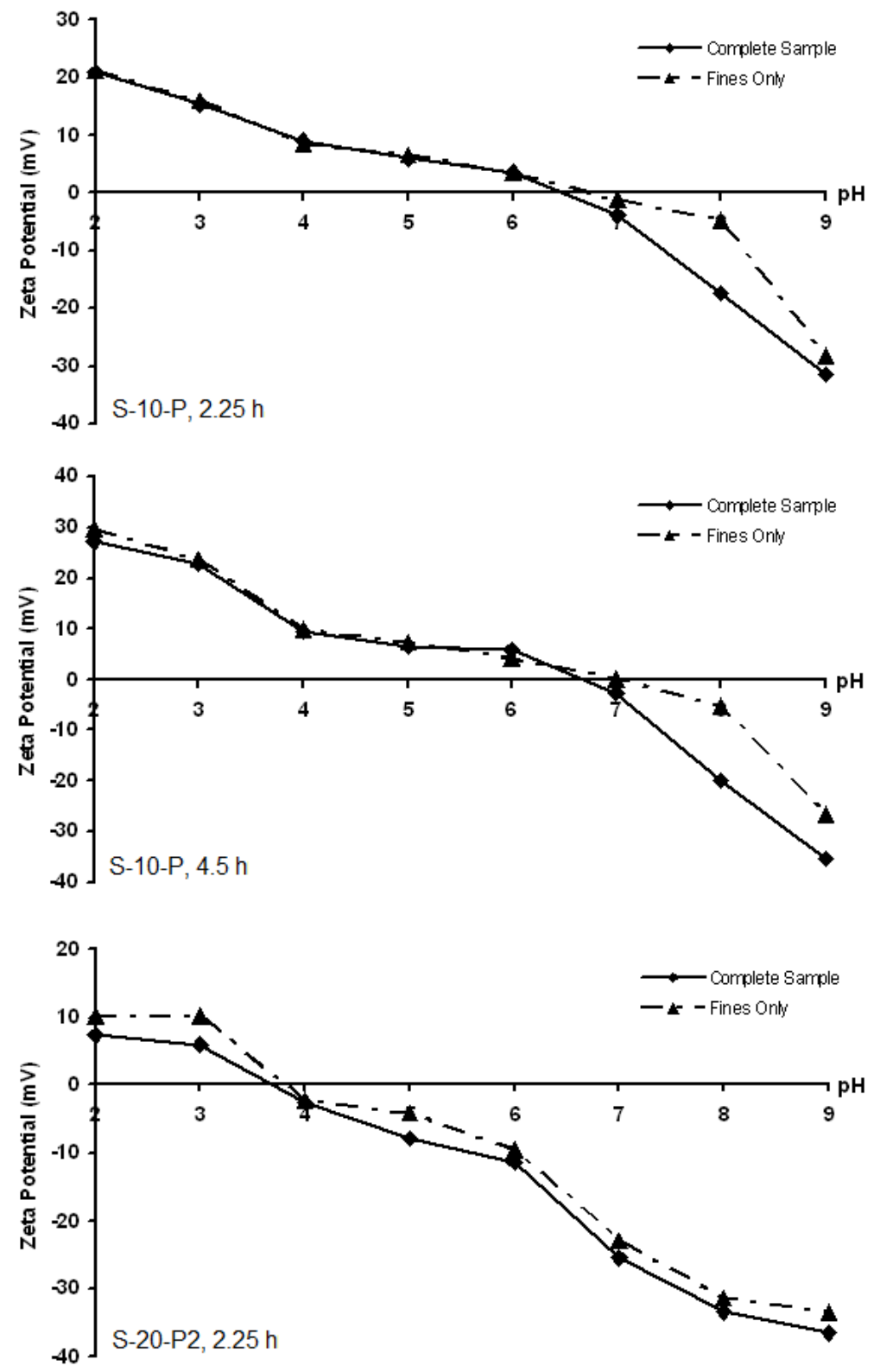

Figure 4: Measured zeta potentials from both bulk and fines samples of S-10-P and S-20-P2, taken from various sampling times (total experiment time is $4.5 \mathrm{~h}$ ) during the respective co-precipitation reactions.

The surface area data presented in Table 3 displays the increase in surface area with increasing Si:Fe ratios. An increase in adsorption would be expected, and the decrease in IEP should favour greater zinc losses due to adsorption. There is likely to be no 
significant effect on the adsorption of anionic impurities; while the surface area is increasing, the affinity for the adsorption of such species is high and would remain so.

The surface area increase at higher Si contents is more prominent in samples prepared in nitrate media, which is due to a combination of two phenomena. The first is that silica polymerisation was not induced in the feed solution, which would ensure that the silica is predominantly surface associated. The second is that nitrate does not interfere with silicic acid adsorption and much greater proportions of silica are precipitated in that anionic environment.

\subsection{Mechanism of Formation}

The combination of XRD, elemental analysis and some physical measurements has led to the development of a formation mechanism involving silica polymerisation prior to ferrihydrite precipitation. Figure 5 displays an illustrated description of the formation process of co-precipitates in both the case of (a) monomeric and (b) polymerised silica. In the case of low or no silica supersaturation (figure 5a), silicate molecules are present in solution as monosilicic acid $\left(\mathrm{Si}(\mathrm{OH})_{4}{ }^{-}\right)$(Swedlund, 1999). Ferrihydrite particles nucleate almost immediately upon the introduction of hydroxide. Once these particles form, silicic acid molecules adsorb on their surface, limiting primary particle growth by populating binding sites. The ferrihydrite particles grow (limited to smaller particle size than in the absence of silica) and aggregate, and at great enough concentrations, the silica polymerises across the aggregates surface. This produces clusters containing primarily ferrihydrite particles with some silica at the grain boundaries and a silicon-rich layer at the aggregate surface.

The other case involves relatively high silica supersaturation and enough time for silica polymerisation to commence prior to iron hydrolysis (figure 5b). Colloidal particles are used in this description, although oligomeric species are also likely to be present, depending on the degree of polymerisation allowed. Silica particles are formed in solution while ferric ions and some silicic acid remain solubilised. At the introduction of hydroxide ferrihydrite particles nucleate, these grow with little resistance from adsorbed silica species and aggregate, incorporating silica particles in 
the process. This produces mixed aggregates which differ significantly from the coprecipitates described in figure $5 \mathrm{a}$.

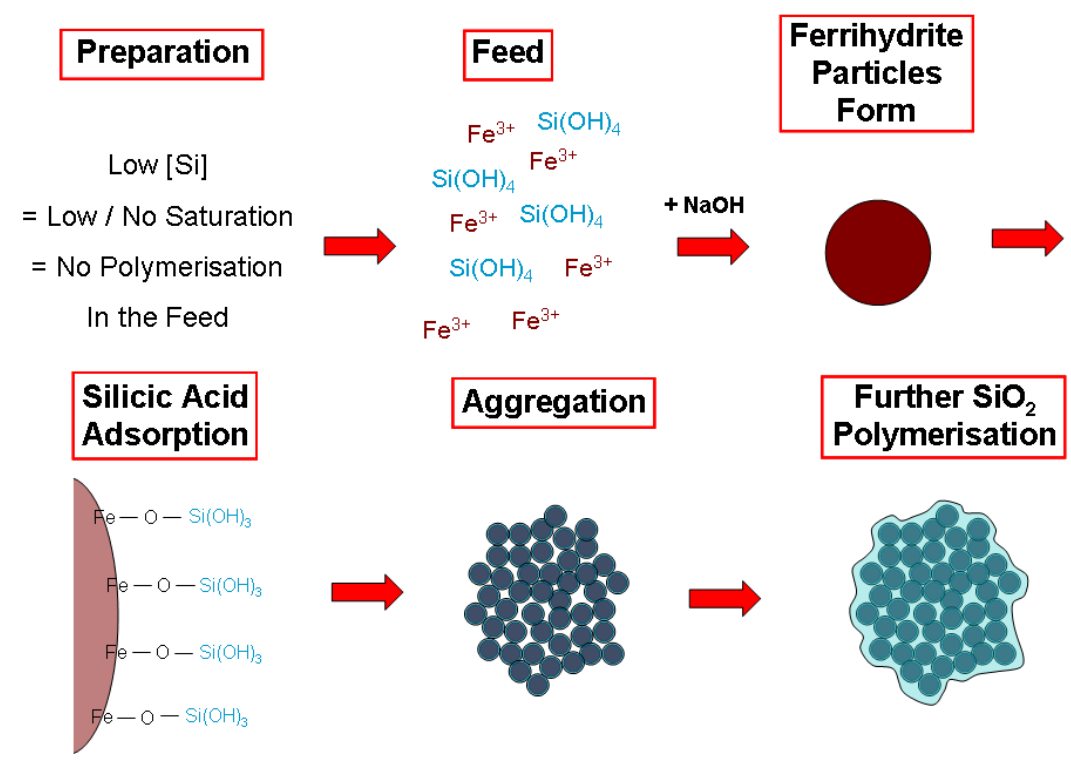

(a)
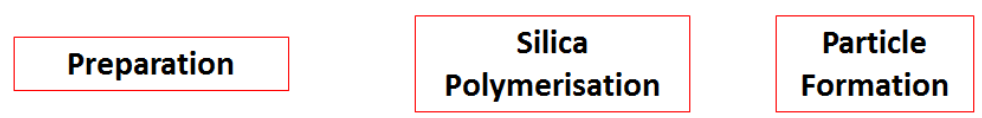

High [Si]

$=$ High Saturation

= Polymerisation

in the Feed

$(\mathrm{HO})_{3} \mathrm{Si}-\mathrm{O}-\mathrm{Si}(\mathrm{OH})_{3}$
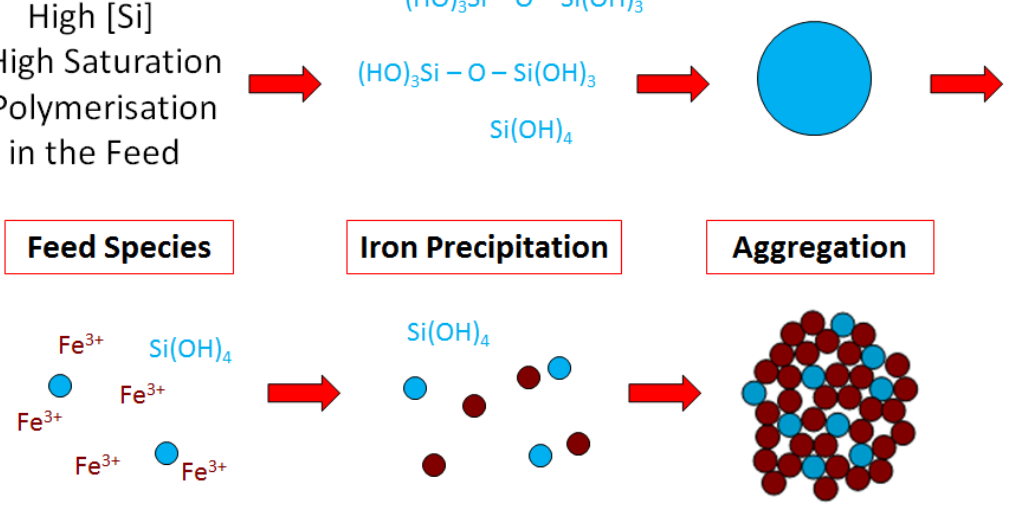

(b)

Figure 5: Illustration depicting the formation mechanism of ferrihydrite/silica co-precipitates in the presence of (a) monomeric and (b) polymeric/colloidal silica.

Process liquors are likely to be supersaturated with respect to silica and, in combination with long time-scales, would favour substantial silica polymerisation to occur. These materials may be more comparable to mixed aggregate composites 
(figure 5b) as opposed to the co-precipitates described in Dyer et al. (Dyer, 2010) (figure 5a), depending on the degree of silica polymerisation.

\section{Conclusions}

Clear evidence has been presented to show that that the polymerisation of silica prior to ferrihydrite precipitation has significant effects on key aspects of the residue properties. As is often the case, there are both positive and negative implications associated with different procedures in residue formation. Therefore conditions should be chosen that lead to a balance of the desired properties. Silica polymerisation in liquors during leaching steps will aid silica removal, but most likely have detrimental effects on the separation characteristics. As managing dewatering properties is a great challenge with regard to the Paragoethite Process as applied in zinc hydrometallurgy, the data presented here would suggest that minimising the degree to which silica polymerises prior to contact with precipitated materials is recommended. Given the longer exposure times in the precipitation reactor and time allowed for further silica adsorption/polymerisation in other tanks post-precipitation, the kinetically slow silica removal may reach a much greater extent in the industrial application than in the experiments detailed in this study.

\section{Acknowledgements}

The work described here has been supported in part by the ARC Linkage project LP 0560753. L. Dyer wishes to acknowledge the Australian Research Council, Nyrstar Metals Ltd., and the Parker Cooperative Research Centre for Integrated Hydrometallurgy Solutions for financial support in the form of a $\mathrm{PhD}$ stipend. Mike Newman and David Palmer are thanked for their advice and comments. Laboratory and analytical support from CSIRO Process Science and Engineering is also acknowledged. 


\section{References}

Arregui, V., Gordon, A. R., Steintveit, G., 1979. Jarosite process - past, present and future. Journal of Metals, 31(12): 22.

Carlson, L., Schwertmann, U., 1981. Natural ferrihydrites in surface deposits from Finland and their association with silica. Geochimica et Cosmochimica Acta, 45: 421-429.

Claassen, J.O., Meyer, E. H. O., Rennie, J., Sadenburgh, R. F., 2002. Iron precipitation from zinc rich solutions: defining the Zincor Process. Hydrometallurgy, 67(1-3): 87-108.

Cooper, R.M.G., 1998. Silica precipitation from electrolytic zinc solutions. Doctoral Thesis, Curtin University of Technology, Perth, Western Australia.

Cornell, R.M., Schwertmann, U., 1996. The Iron Oxides: Structure, Properties, Reactions, Occurrence and Uses. VCH, Weinheim.

Dutrizac, J.E., 1987. An overview of iron precipitation in hydrometallurgy. In: G.L. Strathdee, Klein, M. O., Melis, L. A. (Ed.), International Symposium of Crystallisation and Precipitation. Pergamon Press, Saskatoon, Saskatchewan, Canada, pp. 259.

Dyer, L., Fawell, P. D., Newman, O. M. G., Richmond, R., 2010. Synthesis and characterisation of ferrihydrite/silica co-precipitates. Journal of Colloid and Interface Science, 348(1): 65-70.

Dyer, L.G., Chapman, K. W., English, P., Saunders, M., Richmond, W.R., 2012. Insights into the crystal and aggregate structure of $\mathrm{Fe}^{3+}$ oxide/silica coprecipitates. American Mineralogist, 97.

Fawell, P.D., Owen, A. T., Grabsch, A. F., Benn, F. A., Labbett, D. M., Swift, J. D., 2009. Factors affecting flocculation within gravity thickeners, Water in Mining. The Australian Institute of Mining and Metallurgy., Perth, Western Australia, pp. 71-76.

Jullien, R., Botet, R., 1987. Aggregation and fractal aggregates. World Scientific Publishing, Singapore.

Kershaw, M.G., 1995. Modernisation of the leaching circuit at pasminco metals - EZ. Hydrometallurgy, 39(1-3): 129-145.

Loan, M., 2004. The paragoethite process: Fundamentals of ferrihydrite, schwertmannite and goethite precipitation. Doctoral Thesis, Curtin University of Technology, Perth, Western Australia.

Loan, M., Newman, O. M. G., Cooper, R. M. G., Farrow, J. B., Parkinson, G. M., 2006a. Defining the paragoethite process for iron removal in zinc hydrometallurgy. Hydrometallurgy, 81(2): 104-129.

Loan, M., Newman, O. M. G., Farrow, J. B., Parkinson, G. M., 2006b. Continuous reactive crystallisation of nanoscale six-line ferrihydrite. Crystal Growth and Design, 6(1): 79-86.

Meakin, P., 1988. Fractal Aggregates. Advances in Colloid and Interface Science, 28: 249-331.

Owen, A.T., Fawell, P. D., Swift, J. D., Labbett, D. M., Benn, F. A., Farrow, J. B., 2008. Using turbulent pipe flow to study the factors affection polymerbridging flocculation of mineral systems. International Journal of Mineral Processing, 87: 90-99. 
Richmond, W., Loan, M., Morton, J., Parkinson, G. M., 2004. Arsenic removal from aqueous solution via ferrihydrite crystallisation control. Environmental Science and Technology, 38(12): 2368-2372.

Schwertmann, U., Cornell, R. M., 2000. Iron Oxides in the Laboratory: Preparation and Characterisation. Wiley-VCH, Weinheim.

Selomulya, C., Amal, R., Bushell, G., Waite, T. D., 2001. Evidence of shear rate dependence on restructuring and breakup of latex aggregates. Journal of Colloid and Interface Science, 236: 67-77.

Sigg, L., Stumm, W., 1981. The interaction of anions and weak acid with the hydrous goethite (alpha-FeOOH) surface. Colloids and Surfaces, 2: 101-117.

Swedlund, P.J., Webster, J.G., 1999. Adsorption and polymerisation of silicic acid on ferrihydrite, and its affect on arsenic adsorption. Water Research, 33(16): 3413-3422.

Takemori, M., 1993. Hydrometallurgical methods for extraction and purification of silica. Minerals Engineering, 6(3): 279-290.

Zhou, Y., Franks, G. V., 2006. Flocculation mechanism induced by cationic polymers investigated by light scattering. Langmuir, 22: 6775-6786. 